\title{
Reforming the approach of the Global Environmental Facility to biodiversity conservation
}

\author{
Russell A. Mittermeier and Ian A. Bowles
}

\begin{abstract}
Biodiversity - a measure of the wealth of species, ecosystems and ecological processes that make up our living planet-received public prominence as a result of the Earth Summit in Rio de Janeiro in June 1992. The loss of biodiversity, say the authors, is the greatest environmental problem the world faces but the issue has not been given the attention it deserves. With the emergence of the Global Environmental Facility in 1990 came the chance to fund biodiversity conservation on a unprecedented scale and in 1992 the GEF was adopted as the interim funding mechanism for the Convention on Biological Diversity signed at the Earth Summit. Three years after its foundation, the authors of this paper suggest that the $G E F$ has to be reformed radically if it is to become an effective force in conservation. Their conclusions are based on Conservation International's experience with the GEF over the last 3 years in more than 10 countries.
\end{abstract}

\section{Introduction}

Our planet faces an array of environmental problems, from pollution and soil erosion to climate change and ozone layer depletion, coupled with explosive human population growth in the tropics and excessive resource consumption in developed countries. However, we believe that one issue - the loss of our planet's biological diversity - surpasses all others in terms of long-term global impact.

We now face a series of potential, and possibly already ongoing, extinction spasms unlike anything since the disappearance of the dinosaurs 65 million years ago (see, for example, Myers, 1984; Wilson, 1988, 1992). We are at risk of losing not just single species or communities of different species, but entire ecosystems.

\section{Development of the Global Environment Facility}

Over the last 5 years, we have seen increasing international attention paid to biodiversity.
This culminated, at the UN Conference on Environment and Development (UNCED; the Earth Summit) in June 1992, with the completion of Agenda 21 and a major international Convention on Biological Diversity, which was signed by 165 nations. Concurrent with the Convention negotiations was the development of a global fund for biodiversity conservation and other environmental priorities, the Global Environment Facility (GEF).

The GEF roots go back to the 1987 report of the World Commission on Environment and Development, or Brundtland Commission, which concluded that there was 'a serious lack of funding for conservation projects and strategies that improve the resource base for development' and suggested that 'serious consideration should be given to the development of a special banking program, or facility' to fund conservation projects (WRI, 1989). The United Nations Development Programme (UNDP) then commissioned the World Resources Institute (WRI) to undertake a yearlong study to develop recommendations for new directions in international conservation financing. 
Chief among the new proposals of the WRI (1989) report was the concept of creating International Environmental Facilities to identify and support promising conservation projects. The study envisioned a series of such facilities to play a brokering role between governments, bilateral aid agencies, multilateral development banks (MDBs), intergovernmental organizations and non-governmental organization (NGO) project implementers. In September 1989, the French and German governments proposed the creation of a global ' $\mathrm{fa}$ cility' for this purpose.

The GEF was subsequently created in November 1990 as a collaborative venture of two main implementing agencies, the World Bank and UNDP, with the United Nations Environment Programme (UNEP) serving as the secretariat for the GEF's Scientific and Technical Advisory Panel (STAP). The GEF is chaired by Mohamed El-Ashry, Director of the World Bank's Environment Department, where the GEF's Administration is also housed. From the outset, biodiversity conservation was one of the four principal activities the GEF was to address, the others being global climate change, protection of international waterways and reduction of ozonedepleting chemicals. The initial funding level for the GEF was put at Special Drawing Rights 1 billion (\$US1.3 billion) and to date the GEF core fund has some \$US860 million in commitments. Although only approximately 40 per cent of that is to be spent on biodiversity, it is a sum far beyond the most optimistic imaginings of those who had worked in biodiversity conservation for the past few decades.

The GEF was originally intended to fill an unoccupied niche and facilitate support for initiatives that other donors were not supporting. With the Earth Summit in Rio, the prominence of the GEF grew still more and it was designated the interim funding mechanism for the Convention on Biological Diversity, a move that introduced a new level of political complexity. Nevertheless, the biodiversity portion of the GEF is the largest commitment ever made to this issue by the international community, and it generated immense expectations and optimism (tempered with consid- erable doubt and caution, given the environmental track record of its implementing agencies).

Now, 3 years after the creation of the GEF and the approval of the First Tranche of projects (May 1991) and at the end of its Pilot Phase, it is critical to assess the effectiveness of the GEF's biodiversity component. The purpose of this paper is to do just that. We believe that the GEF still has immense potential to effect change and to turn the tide on this critically important issue, but we also believe that major reforms are needed if the GEF is to have real impact on global biodiversity.

\section{Suggestions for reform of the GEF biodiversity portfolio}

Since its creation in November 1990, the GEF has assembled a portfolio of some 44 biodiversity projects with a total value of approximately $\$ 300$ million. This accounts for 43 per cent of the total GEF work programme. Projects range in cost from \$1-3 million in nations such as Guyana, Cuba and the Seychelles to \$25-30 million in Brazil and Mexico (GEF, 1992).

By mid-1994 the GEF plans to have completed its self-evaluation and restructuring and secured financial replenishment. Having received the necessary 30 ratifications, the Convention on Biological Diversity entered into legal force in late 1993. High on the agenda of the first Conference of the Parties, to be held in late 1994, will be its future financial mechanism. The final status of the GEF in relation to the Convention and in general, probably depends on the reforms adopted and implemented prior to that time.

Below we identify some of the problems with the biodiversity component of the GEF, based on our experience to date and make recommendations as to how it could be dramatically improved and turned into a major positive force in global biodiversity conservation.

\section{Niche and mission}

What is the niche that the GEF seeks to fill in 
the area of biodiversity conservation? At present it is a loose aggregate of activities, a set of projects that are really a variety of experiments, some piggybacked on existing World Bank or UNDP projects and others standing alone. The GEF project portfolio reflects its poorly articulated mission, and a shifting set of objectives that has resulted from the political and institutional demands of its many constituencies.

In general, international funding for biodiversity conservation thus far has come from bilateral donors, specialized inter-governmental organizations and international NGOs and their foundation partners. The GEF must improve greatly its collaboration with and, at the same time, differentiate itself from these existing channels. Otherwise donor governments will probably choose their own bilateral donors for assisting with biodiversity conservation.

One problem with the GEF's approach to date is that it may do more harm than good. For example, the GEF's entry into biodiversity conservation has caused some other donors (e.g. several major US charitable foundations) to consider reducing their own levels of support. Because of the operational culture of the GEF Implementing Agencies, there may be an overall reduction in small-scale, NGO or community-based projects, which many have found to be the most effective for conserving biodiversity. The broader result may be a missed opportunity for the GEF to fulfil its original mission of finding potential donors to fund key conservation projects.

The large size of the GEF projects and relative inexperience of the GEF Implementing Agencies has created another unfortunate byproduct in several countries. The GEF has created high expectations, most of which have not been met. These expectations and the large size of promised or perceived GEF funding has in many cases brought about intense infighting and jockeying among both the governmental and NGO communities in many countries as different players seek to gain access to GEF funds. The GEF must take a hard look at its niche with regard to other players and define a mission that plays on the strength of its Implementing Agencies without undermining the ongoing work of bilateral agencies, NGOs, and charitable foundations that support biodiversity conservation.

\section{Incremental costs}

The GEF was designed in part to carry out the funding provisions of the Rio treaties on climate change and biodiversity. Its specific mission was to fund the 'incremental cost' of achieving a global conservation benefit by implementing the treaties. In other words, while the recommendations of Agenda 21 were to address current and future development expenditures, the GEF was to provide the additional funds above and beyond current expenditures to allow developing nations to provide a global conservation benefit that may not be 'in their immediate national interest'.

It is our view that this explanation of incremental costs reflects a basic misunderstanding of the full range of biodiversity values. One can argue that global biodiversity ought to be conserved for its carbon sequestration function or its future biotechnological potential, but that misses most of the benefits that maintaining biodiversity provides. Use of biological resources provides national, regional and local benefits, not in the future, but right here and now.

The GEF's philosophical framework relating to incremental costs is therefore at cross purposes with the overall goal of biodiversity conservation and winds up perpetuating the belief, both in the World Bank and other international institutions and in developing countries themselves, that biodiversity is not fundamental to a nation's economic viability. Nothing could be further from the truth. The full range and value of current uses of biological resources at all levels need to be recognized by the GEF and used as a basic principle in all of its activities.

In our view, the notion of incremental costs may apply to global climate change or to ozone depletion (although even this is being debated), but it does not fit at all with biodiversity conservation. This fundamental philosophical problem with the GEF must be ad- 
dressed if it is to have a positive impact on biodiversity conservation. Indeed, the GEF should be at the forefront of a movement to integrate biodiversity into the mainstream of the development process, rather than perpetuating a philosophy that ensures its continued marginalization. The GEF should serve as a vehicle to bring about basic change in its Implementing Agencies, and, through its influence with donor and recipient nations, change the way the world looks at biodiversity values.

\section{Project cycle}

The need for resources for biodiversity conservation is immediate, especially in the highest priority areas at greatest risk. However, it is also a long-term endeavour that requires a sustained, yet flexible, source of funding. The current GEF project cycle is too long and cumbersome to deal effectively with the urgency of this issue and lacks the agility needed to respond to the ever-changing needs of biodiversity conservation. While the World Bank and its project cycle may be well suited to developing and managing large-scale climate-change projects that concern the entire energy sector of a given country, they are poorly suited to biodiversity conservation. Most of the expertise on biodiversity and the capacity to implement field projects rests outside the government sector, and requires small but rapidly disbursed funding with some measure of continuity beyond the initial GEF project period.

In its first 2 years, out of a work programme in excess of $\$ 700$ million, the GEF has disbursed only $\$ 2.8$ million to actual biodiversity projects, while spending more than $\$ 20$ million on administrative expenses (GEF, 1992). The GEF has also been constrained by its requirement to channel funds only through central governments. Both are in part a reflection of the nature of the Implementing Agencies of the GEF. Biodiversity conservation requires a more flexible and streamlined approach, with a much shorter project cycle and smaller initial investments with a mechanism to ensure long-term funding.

Project review should also be placed more in the hands of national or international NGOs with long-term experience in a country or project site. The use of consultants is acceptable if they have relevant experience, but very often consultants used to review projects know little or nothing of the areas they are sent to evaluate. The results are sometimes ludicrous. For example, a consultant sent to assess priorities in Brazil recommended investments in two of the biologically least important regions in the country (G. Fonseca, pers. comm.). This is particularly egregious in a country where priorities are reasonably well established and understood.

\section{Project size}

The absorptive capacity of the natural resources management sector of most GEF recipient countries is very limited. None the less, many GEF projects propose to flood millions of dollars into sectors that are unable to use the money effectively over the short periods of most projects designed to date. Indeed, we fear that the tendency towards large, single-shot investments by the GEF will create overnight institutions that are likely to collapse when the international donor community turns its attention elsewhere.

Rather than requiring a lengthy approval process for a single $\$ 10$ million GEF project, we would prefer to see, for example, $10 \$ 1$ million projects, $20 \$ 500,000$ projects or even $200 \$ 50,000$ projects spread out over several years. A diversified approach would have multiple benefits. It would allow the GEF to build both governmental and non-governmental capacity, it would allow for smaller, more flexible projects, it would reduce destructive jockeying and political infighting, and it would make use of the real expertise that exists.

The GEF Small Grants Programme for NGOs is a good first step, but it represents only some 2-3 per cent of the total GEF portfolio. It should be expanded in size and grettly streamlined, but it is just one part of the kind of approach that is needed. The GEF should adopt a much more flexible funding approach that relies primarily on small- to medium- 
sized grants to governments and NGOs alike, and incorporates mechanisms (for example, trust funds and endowments) that ensure some continuity of support beyond the 3-5 year time span of most GEF projects.

\section{Trust funds}

With the GEF's emphasis on innovation, institution-building and cost-effectiveness as well as the pressing need for continuity of investments in biodiversity projects, the use of trust funds and endowments for biodiversity conservation seems a perfect match. Hard-currency trust funds, for example, not only provide a hedge against currency devaluation but also help to address our concerns about project size, project cycle, long-term funding and emphasis on local capacity-building. The GEF has already used this approach in Bhutan, Peru and several other countries. We recommend that it be considered in many other biodiversity projects as well.

\section{Use of NGOs}

Unlike global climate change, expertise in biodiversity conservation lies principally with local, national and international NGOs. The GEF must greatly enhance its efforts to involve NGOs and other civil sector participation in its biodiversity projects. Field-based NGOs in particular offer a wealth of technical expertise and local knowledge. It should become standard practice for the GEF to seek the advice of local, national and international field-based NGOs in project design and implementation.

In our experience, the GEF has sought input from NGOs, but thus far mainly in a piecemeal fashion that, in some cases, has resulted in infighting, rather than collaboration. The GEF should consider greater partnerships with structures like the IUCN Species Survival Commission, which has a volunteer network of more than 5000 biodiversity specialists from developing and developed countries alike, and represents the single largest body of expertise in the world on this issue.

\section{Priority setting}

The GEF needs to recognize that distribution of the world's biological resources is very uneven, with certain areas having far higher concentrations of diversity and suffering far greater pressures. It should set priorities for action, rather than reacting only to political pressures or responding to projects on a firstcome, first-served basis. To do this will require developing a mechanism that incorporates the priority-setting expertise of key NGOs and tailor them to the needs of the GEF (while recognizing that some degree of political accommodation will always be necessary).

One such mechanism might be the Global Biodiversity Assessment currently being implemented by the GEF and UNEP. Building on background documents such as the Global Biodiversity Strategy (WRI et al., 1992) and the Global Biodiversity Status Report (WCMC, 1992), such a structure could incorporate the priority-setting expertise of NGOs that have focused on this issue and come up with the most appropriate course for further GEF action.

The GEF should quickly signal its intent to create a true partnership with the array of NGOs that have taken a serious look at biodiversity conservation priorities, and form a more technically robust secretariat to process this critical information and complete a global assessment of our knowledge of biodiversity. After over a decade of neglect, we are finally seeing political support in the United States for a national Biological Survey to create a greater scientific basis for conservation and sustainable use of natural resources. Mexico, Australia and several other tropical countries have begun similar exercises. The GEF is uniquely positioned to broker an international equivalent to help better define global biodiversity priorities.

\section{Institutional considerations}

Recent history has seen a welcome trend toward democracy and public participation. The UNCED process afforded NGOs and the public unparalleled access to information and par- 
ticipation in the policy-making process. The GEF should make a greater effort to build on the UNCED model and hold public consultations with communities and constituencies likely to be affected by projects (a suggestion that applies not only to the GEF, but the international development process as a whole).

Although much of development assistance is theoretically aimed at alleviating poverty, it has in the past often exacerbated the problem because the intended beneficiaries were not involved in programme design and implementation. Transparency and information disclosure are critical parts of building public trust. The MDBs have a particularly poor track record in this regard. The GEF will become more effective and will be more respected if it provides public information at all stages of a project cycle. Biodiversity conservation is by its very nature a participatory and local endeavour; it cannot be brought about without the support of a diverse set of interests, beginning with the local people. To be effective and become a politically viable institution, the GEF must make fundamental changes in its operating procedures to take this into account.

The GEF would also gain greater credibility by creating an independent secretariat. It was originally conceived as an organization that would act as a broker to identify high-priority conservation projects and potential funders for those projects. It would be more effective as such a facilitator of diversified funding approach, one that uses the strengths of the MDBs, bilateral donors, UN agencies, specialized intergovernmental organizations and the range of NGO project implementers, if its secretariat has a measure of independence from its current Implementing Agencies.

\section{Conclusion}

Biodiversity conservation is the one major environmental challenge that cannot be addressed through technological innovation alone. In the GEF, the international community, for the first time, has significant financial resources available to conserve the poorly understood, fundamentally important living natural resource base. Now, more than 3 years after its creation, valuable lessons have emerged, and these should be used to design a far more effective structure for future biodiversity conservation efforts.

More than anything else, the role of the GEF in biodiversity conservation depends on a clear definition of mission and niche, some distancing from the approaches and philosophies of its Implementing Agencies, greatly enhanced collaboration with the NGO sector, and the assumption of a clear leadership on this issue within the international development community.

The opportunity to effect major change clearly exists, but it will require a dynamic process of introspection and restructuring to be truly successful. In years hence, the GEF could be looked upon as the institution that turned the tide in efforts to maintain the diversity of life on Earth; or it could be counted as just one more in an endless string of wellintentioned but essentially fruitless development programmes so characteristic of the latter half of the twentieth century.

\section{References}

Global Environment Facility. 1992. Report by the Chairman to the December 1992 Participants Meeting. World Bank, Washington, DC.

Myers, N. 1984. The Primary Source: Tropical Forests and Our Future. W.W. Norton and Co., New York.

Wilson, E.O. (ed.) 1988. Biodiversity. National Academy Press, Washington, D.C.

Wilson, E.O. 1992. The Diversity of Life. Harvard University, Cambridge, MA.

World Conservation Monitoring Centre. 1992. Global Biodiversity: Status of the Earth's Living Resources. WCMC, Cambridge, UK.

WRI. 1989. Natural Endowments: Financing Resource Conservation for Development. World Resources Institute, Washington, DC.

WRI, IUCN, UNEP. 1992. Global Biodiversity Strategy: Guidelines for Action to Save, Study, and Use the Earths Biotic Wealth Sustainably and Equitably. World Resources Institute, Washington, DC.

Russell A. Mittermeier and Ian A. Bowles, Conservation International, 1015 18th St, NW, Suite 1000, Washington, DC 20036, USA. 